\title{
Ball and Spindle Convexity with respect to a Convex Body
}

\author{
Zsolt Lángi \\ Dept. of Geometry, Budapest University of Technology and Economics, Egry József u. 1, \\ Budapest, Hungary, 1111 \\ Márton Naszódi ${ }^{1}$ \\ Dept. of Geometry, Eötvös University, Pázmány Péter s. 1/c, Budapest, Hungary, 1117 \\ István Talata ${ }^{2}$ \\ Dept. of Geometry and Informatics, Ybl Faculty of Architecture and Civil Engineering, \\ Szent István University, Thököly út 74, Budapest, Hungary, 1146
}

\begin{abstract}
Let $C \subset \mathbb{R}^{n}$ be a convex body. We introduce two notions of convexity associated to C. A set $K$ is $C$-ball convex if it is the intersection of translates of $C$, or it is either $\emptyset$, or $\mathbb{R}^{n}$. The $C$-ball convex hull of two points is called a $C$-spindle. $K$ is $C$-spindle convex if it contains the $C$-spindle of any pair of its points. We investigate how some fundamental properties of conventional convex sets can be adapted to $C$-spindle convex and $C$-ball convex sets. We study separation properties and Carathéodory numbers of these two convexity structures. We investigate the basic properties of arc-distance, a quantity defined by a centrally symmetric planar $\operatorname{disc} C$, which is the length of an arc of a translate of $C$, measured in the $C$-norm, that connects two points. Then we characterize those $n$-dimensional convex bodies $C$ for which every $C$-ball convex set is the $C$-ball convex hull of finitely many points. Finally, we obtain a stability result concerning covering numbers of some $C$-ball convex sets, and diametrically maximal sets in $n$-dimensional Minkowski spaces.
\end{abstract}

Email addresses: zlangi@math.bme.hu (Zsolt Lángi), nmarci@math.elte.hu (Márton Naszódi), talata.istvan@ybl.szie.hu (István Talata)

${ }^{1}$ Partially supported by the Hung. Nat. Sci. Found. (OTKA) grant no. K72537.

${ }^{2}$ Partially supported by the Hung. Nat. Sci. Found. (OTKA) grant no. K68398. 
Keywords: ball convexity, spindle convexity, ball-polyhedron, separation, Carathéodory's theorem, convexity structure, illumination, arc-distance 2008 MSC: 2A30, 2008 MSC: 52A35, 2008 MSC: $52 \mathrm{C} 17$,

\section{Introduction}

Closed convex sets may be introduced in two distinct manners: either as intersections of half-spaces, or as closed sets which contain the line segments connecting any pair of their points. We develop these approaches further to obtain the notions of ball convexity and spindle convexity with respect to a convex body. Let $C$ be a convex body (a compact convex set with non-empty interior) in Euclidean $n$-space, $\mathbb{R}^{n}$.

Definition 1.1. A set $K \subset \mathbb{R}^{n}$ is called ball convex with respect to $C$ (shortly, $C$-ball convex), if it is either $\emptyset$, or $\mathbb{R}^{n}$, or the intersection of all translates of $C$ that contain $K$.

Definition 1.2. Consider two (not necessarily distinct) points $p, q \in \mathbb{R}^{n}$ such that there is a translate of $C$ that contains both $p$ and $q$. Then the $C$-spindle (denoted as $[p, q]_{C}$ ) of $p$ and $q$ is the intersection of all translates of $C$ that contain $p$ and $q$. If no translate of $C$ contains $p$ and $q$, we set $[p, q]_{C}=\mathbb{R}^{n}$. We call a set $K \subset \mathbb{R}^{n}$ spindle convex with respect to $C$ (shortly, $C$-spindle convex), if for any $p, q \in K$, we have $[p, q]_{C} \subset K$.

In this paper, we study the geometric properties of ball and spindle convex sets depending on $C$, and how these two families are related to each other.

In 1935, Mayer [26] defined the notion of "Überkonvexität". His definition coincides with our spindle convexity with the additional assumption that $\mathrm{C}$ is smooth and strictly convex.

Intersections of (finitely many) Euclidean unit balls on the plane were studied by Bieberbach [7], and in three dimensions by Heppes [13], Heppes and Révész [14], Straszewitcz [35] and Grünbaum [12]. Recent developments have led to further investigations of sets that are ball convex with respect to the Euclidean unit ball $B^{n}$. For these results, the reader is referred to [3], [6], [8], [19], [18], [17] and [28]. A systematic investigation of these sets was started by Bezdek et al. [5]: the authors examined how fundamental properties of convex sets can be transferred to sets that are ball convex 
with respect to $B^{n}$; in particular, they gave analogues of Kirchberger's and Carathéodory's theorems, examined separation properties of ball convex sets and variants of the Kneser-Poulsen conjecture. It is also shown there that the notions of ball and spindle convexity coincide when $C=B^{n}$. In the spirit of the results in [5], the authors of [20] gave generalizations of Kirchberger's theorem for separation of finite point sets by homothets or similar copies of a given convex region. The notion of $C$-ball convex hull (cf. Definition 2.2) was defined for centrally symmetric plane convex bodies by Martini and Spirova [26].

In Section 2, we introduce our notation and basic concepts. In Section 3, we examine how the notions of ball and spindle convexity are related to each other and how sets are separated by translates of $C$. In Section 4, we define the arc-distance between two points with respect to a planar disc $C$, and examine when the triangle inequality holds and when it fails. In Section 5, we introduce and examine the Carathéodory numbers associated to these convexity notions. In Section 6 , we give a partial characterization of convex bodies $C$ for which every $C$-ball convex set is the $C$-ball convex hull of finitely many points. Finally, in Section 7 , we prove that the operation of taking intersections of translates of $C$ is stable in a certain sense. By applying this result to Hadwiger's Covering Problem for certain $C$-ball convex sets, and diametrically maximal sets in a Minkowski space, we obtain a stability of upper bounds on covering numbers.

\section{Spindle and ball convex hull, convexity structures}

We use the standard notation bd $S, \operatorname{int} S, \operatorname{relint} S$, aff $S, \operatorname{conv}(S)$ and card $S$ for the boundary, the interior, the relative interior, the affine hull, the (linear) convex hull and the cardinality of a set $S$ in $\mathbb{R}^{n}$. For two points $p, q \in \mathbb{R}^{n},[p, q]$ denotes the closed segment connecting $p$ and $q$. The vectors $e_{1}, e_{2}, \ldots, e_{n} \in \mathbb{R}^{n}$ denote the standard orthonormal basis of the space, and for a point $x \in \mathbb{R}^{n}$, the coordinates with respect to this basis are $x=\left(x_{1}, x_{2}, \ldots, x_{n}\right)$. The Euclidean norm of $p \in \mathbb{R}^{n}$ is denoted by $|p|$. As usual, $\alpha A+\beta B$ denotes the Minkowski combination of sets $A, B \subset \mathbb{R}^{n}$ with coefficients $\alpha, \beta \in \mathbb{R}$ (cf. [31]).

Definition 2.1. Let $C \subset \mathbb{R}^{n}$ be a convex body, $X \subseteq \mathbb{R}^{n}$ a nonempty set and $r \geq 0$. Then we set

$$
\mathbf{B}_{C}^{+}(X, r)=\bigcap_{v \in X}(r C+v) \quad \text { and } \quad \mathbf{B}_{C}^{-}(X, r)=\bigcap_{v \in X}(-r C+v)
$$


Furthermore, we set $\mathbf{B}_{C}^{+}(\emptyset, r)=\mathbf{B}_{C}^{-}(\emptyset, r)=\mathbb{R}^{n}$. When $r$ is omitted, it is one: $\mathbf{B}_{C}^{+}(X)=\mathbf{B}_{C}^{+}(X, 1), \mathbf{B}_{C}^{-}(X)=\mathbf{B}_{C}^{-}(X, 1)$. When $C=-C$ we may omit the $+/-$ signs.

Note that by Definition 1.2, we have

$$
[p, q]_{C}=\mathbf{B}_{C}^{+} \mathbf{B}_{C}^{-}(\{p, q\})
$$

In the paper we often use the following two fundamental concepts.

Definition 2.2. The spindle convex hull of a set $A$ with respect to $C$ (in short, $C$-spindle convex hull), denoted by $\operatorname{conv}_{C}^{s}(A)$, is the intersection of all sets that contain $A$ and are spindle convex with respect to $C$. The ball convex hull of $A$ with respect to $C$ (or $C$-ball convex hull), denoted by $\operatorname{conv}_{C}^{b}(A)$, is the intersection of all $C$-ball convex sets that contain $A$.

We remark that $\operatorname{conv}_{C}^{b}(A)$ is the intersection of those translates of $C$ that contain $C$, or in other words, $\operatorname{conv}_{C}^{b}(A)=\mathbf{B}_{C}^{+} \mathbf{B}_{C}^{-}(A)$.

Next, we study the notions of ball and spindle convexity in the context of the theory of abstract convexity.

Definition 2.3. A convexity space is a set $X$ together with a collection $\mathcal{G} \subseteq \mathcal{P}(X)$ of subsets of $X$ that satisfy

i. $\emptyset, X \in \mathcal{G}$, and

ii. $\mathcal{G}$ is closed under arbitrary intersection.

Such a collection $\mathcal{G}$ of subsets of $X$ is called a convexity structure on $X$. If a third condition

(iii) $\mathcal{G}$ is closed under the union of any increasing chains (with respect to inclusion)

also holds, then we call the pair $(X, \mathcal{G})$ an aligned space (and $\mathcal{G}$ an aligned space structure). This is the terminology used, for example, by Sierksma [34] and by Kay and Womble [15].

We note that in the literature (cf. van de Vel [36]), if $\mathcal{G}$ satisfies (i) and (ii), but does not necessarily satisfy (iii), then it is often referred to as a Moore family, or a closure system. On the other hand, 'convexity space' frequently refers to what we call an aligned space (see [16]). Other terms used for an aligned space in the literature are 'domain finite convexity space' and 'algebraic closure system'. 
The convex hull operation associated with a convexity space $(X, \mathcal{G})$ is: $\operatorname{conv}_{\mathcal{G}}(A)=\cap\{G \in \mathcal{G}: A \subseteq G\}$ for any $A \subseteq X$. The roughest convexity (resp. aligned space) structure $\mathcal{G}$ which contains a given family $\mathcal{S} \subseteq \mathcal{P}(X)$ is the convexity (resp. aligned space) structure generated by $\mathcal{S}$. This is the intersection of all convexity (resp. aligned space) structures which contain $\mathcal{S}$.

For a convex body $C \subseteq \mathbb{R}^{n}$, we denote the family of $C$-ball convex sets by $\mathcal{B}_{C}$. Clearly, $\left(\mathbb{R}^{n}, \mathcal{B}_{C}\right)$ is a convexity space. We note that the family of $C$-spindle convex sets is an aligned space structure, while the family of closed $C$-spindle convex sets is a convexity structure. Furthermore, the space of $C$ spindle convex bodies is a geometrical aligned space (an aligned space $(X, \mathcal{G})$ is called geometrical if $A=\bigcup\left\{\operatorname{conv}_{\mathcal{G}}(F) \mid F \subseteq A, \operatorname{card}(F) \leq 2\right\}$ for every $A \in \mathcal{G}$, see $[16])$.

Clearly, $\mathcal{B}_{C}$ is the convexity space generated by the translates of $C$. Let $\mathcal{T}_{C}$ denote the aligned space structure generated by these translates. In Theorem 1, we compare their corresponding hull operations $\operatorname{conv}_{C}^{b}$ and $\operatorname{conv}_{\mathcal{T}_{C}}$. For the proof we need the following result of Sierksma [34].

Lemma 2.4 (Theorem 7 in [34]). Let $(X, \mathcal{S})$ be a convexity space, and let $\mathcal{G}$ be the aligned space structure generated by $\mathcal{S}$. If $A \subseteq X$, then

$$
\operatorname{conv}_{\mathcal{G}}(A)=\bigcup_{k=0}^{\infty}\left[\cup\left\{\operatorname{conv}_{\mathcal{S}}(F): F \subseteq A,|F| \leq k\right\}\right]
$$

Theorem 1. Let $C$ be a convex body in $\mathbb{R}^{n}$, and let $A \subseteq \mathbb{R}^{n}$. Assume that $\operatorname{dim}_{\operatorname{conv}}^{b}(A)=n$. Then

$$
\operatorname{cl}\left(\operatorname{conv}_{\mathcal{T}_{C}}(A)\right)=\operatorname{conv}_{C}^{b}(A)
$$

that is, $\mathrm{cl}\left(\operatorname{conv}_{\mathcal{T}_{C}}(A)\right)$ is the intersection of all translates of $C$ that contain A.

Proof. Clearly, $\operatorname{cl}\left(\operatorname{conv}_{\mathcal{T}_{C}}(A)\right) \subseteq \mathbf{B}_{C}^{+} \mathbf{B}_{C}^{-}(A)$. To prove the reverse containment, assume that $x \in \operatorname{int} \mathbf{B}_{C}^{+} \mathbf{B}_{C}^{-}(A)$. By Lemma 2.4, it is sufficient to show that $x \in \mathbf{B}_{C}^{+} \mathbf{B}_{C}^{-}(F)$ for some finite $F \subseteq A$.

Since $-\operatorname{int} C+x \supseteq \mathbf{B}_{C}^{-}(A)$ (see Remark 5.5), we have $(-\operatorname{bd} C+x) \cap$ $\mathbf{B}_{C}^{-}(A)=\emptyset$. From the compactness of $-\mathrm{bd} C+x$ it follows that there is an $F \subseteq A$ finite set with $(-\operatorname{bd} C+x) \cap \mathbf{B}_{C}^{-}(F)=\emptyset$. Thus, $x \in \mathbf{B}_{C}^{+} \mathbf{B}_{C}^{-}(F)$. 
Clearly, if any $C$-spindle is $n$-dimensional, then so is the $C$-ball convex hull of any set containing more than one point. This leads to the following observation.

Remark 2.5. If $C$ is a strictly convex body in $\mathbb{R}^{n}$, then $\operatorname{cl}\left(\operatorname{conv}_{\mathcal{T}_{C}}(A)\right)=$ $\operatorname{conv}_{C}^{b}(A)$ for any set $A \subset \mathbb{R}^{n}$.

We expect a positive answer to the following question:

Problem 2.6. Can we drop the condition on the dimension of $\operatorname{conv}_{C}^{b}(A)$ in Theorem 1?

\section{Relationship between spindle and ball convexity, and separation by translates of a convex body}

Clearly, for any convex body $C$, a $C$-ball convex set is closed and $C$ spindle convex. Thus, for any $X$ and $C$ the $C$-ball hull of $X$ contains its $C$-spindle convex hull. In [5], it is shown that if $C$ is the Euclidean unit ball, then for closed sets the notions of spindle and ball convexity coincide. Now we show that it is not so for every convex body $C$.

Example 3.1. We describe a 3-dimensional convex body $C$ and a set $H \subseteq \mathbb{R}^{3}$ for which $H$ is $C$-spindle convex but it is not $C$-ball convex. Let $T \subset \mathbb{R}^{3}$ be a regular triangle in the $x_{3}=0$ plane, with the origin as its centroid (cf. Figure 1). Let $C=\operatorname{conv}\left(\left(T+e_{3}\right) \cup\left(-T-e_{3}\right)\right)$. Let $H$ be the intersection of $C$ with the plane with the equation $x_{3}=0$. Note that $H$ is a regular hexagon: $H=(T-T) / 2$.

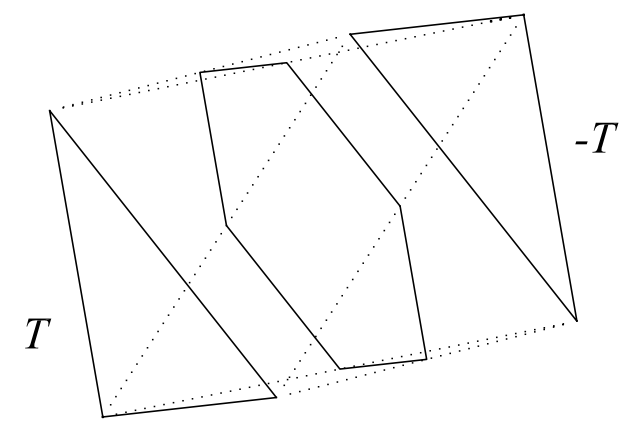

Figure 1: Example 3.1 
We show that $H$ is $C$-spindle convex. Note that $H$ and $T$ are of constant width two in the two-dimensional norm defined by $H$. Thus, for any $p, q \in H$, there is a chord of $T$, parallel to $[p, q]$, that is not shorter than $[p, q]$. From this, it follows that there is a translate $T+z_{1}$ of $T$ that contains $p, q$, and similarly, a set $-T+z_{2}$ containing $p$ and $q$. We observe that $[p, q]_{C} \subseteq$ $C \cap\left(C+z_{1}-e_{3}\right) \cap\left(C+z_{2}+e_{3}\right) \subseteq H$, which yields the desired statement.

We have that $\operatorname{conv}_{C}^{s}(H)=H$ but clearly, there is only one translate of $C$ containing $H$, namely $C$. Thus, $H=\operatorname{conv}_{C}^{s}(H) \neq \operatorname{conv}_{C}^{b}(H)=C$.

We introduce the following notions. Note the order of $X$ and $Y$ in Definition 3.2 .

Definition 3.2. Let $C \subset \mathbb{R}^{n}$ be a convex body, and let $X, Y \subset \mathbb{R}^{n}$. We say that a translate $C+x$ of $C$ separates $X$ from $Y$, if $X \subset C+x$, and $\operatorname{int}(C+x) \cap Y=\emptyset$. If $X \subset \operatorname{int}(C+x)$ and $(C+x) \cap Y=\emptyset$, then we say that $C+x$ strictly separates $X$ from $Y$.

In [5], it is proved that if $C=B^{n}$ is the Euclidean ball, then any $C$ spindle convex set is separated from any non-overlapping $C$-spindle convex set by a translate of $C$. By Example 3.1, not all convex bodies have this property (there, $H$ is not separated from any singleton subset of $C$ ). Thus, we introduce the following notions.

Definition 3.3. Let $C \subset \mathbb{R}^{n}$ be a convex body, and let $K \subset \mathbb{R}^{n}$ be a $C$ spindle convex set. We say that $K$ satisfies Property (p), (s) or (h) with respect to $C$, if

(p) For every point $p \notin K$, there is a translate of $C$ that separates $K$ from $p$.

(s) For every $C$-spindle convex set $K^{\prime}$ that does not overlap with $K$ there is a translate of $C$ that separates $K$ from $K^{\prime}$.

(h) For every hyperplane $H$ with $H \cap$ int $K=\emptyset$, there is a translate of $C$ that separates $K$ from $H$.

It is not difficult to show that Property (h) yields (s), which in turn yields (p).

Remark 3.4. A closed set $K$ satisfies ( $p)$ if, and only if, $K$ is $C$-ball convex. In particular, for closed sets the notions of spindle convexity and ball convexity with respect to a convex body $C$ coincide if, and only if, every closed $C$-spindle convex set satisfies ( $p$ ). 
Remark 3.5. For a smooth convex body $C$, (s) and (h) are equivalent.

Recall that in Example 3.1, $H$ is $C$-spindle convex but not $C$-ball convex. We note that $C$ may be replaced by a smooth $C^{\prime}$ such that $H$ is $C^{\prime}$-spindle convex but not $C^{\prime}$-ball convex. Simply, apply the following theorem for the convex body $C$ of Example 3.1 and any smooth and strictly convex body $D$. Then, it follows that $H$ of Example 3.1 is $C^{\prime}$-spindle convex for $C^{\prime}=C+D$, but it is easy to see that $H$ is not $C^{\prime}$-ball convex.

Theorem 2. Let $C, D$ be convex bodies in $\mathbb{R}^{n}$ and let $S \subseteq \mathbb{R}^{n}$. If $S$ is $C$-ball convex, then $S$ is $(C+D)$-ball convex. Similarly, if $S$ is $C$-spindle convex, then $S$ is $(C+D)$-spindle convex.

We need the following standard lemma, for a proof, see Lemma 3.1.8. in [31].

Lemma 3.6. If $C, D$ are convex bodies in $\mathbb{R}^{n}$, then $C=\bigcap_{x \in D}(C+D-x)$.

Proof of Theorem 2. Observe that by Lemma 3.6 we have that $\operatorname{conv}_{C+D}^{b}(S) \subseteq$ $\operatorname{conv}_{C}^{b}(S)$, and $\operatorname{conv}_{C+D}^{s}(S) \subseteq \operatorname{conv}_{C}^{s}(S)$, for any $S \subseteq \mathbb{R}^{n}$. These readily imply the statement of the theorem concerning ball convexity. The statement concerning spindle convexity follows from the fact that for any two points $p, q \in S$ we have $[p, q]_{C+D}=\operatorname{conv}_{C+D}^{b}(\{p, q\}) \subseteq \operatorname{conv}_{C}^{b}(\{p, q\})=[p, q]_{C}$.

A frequently used special case of Theorem 2 is the following.

Corollary 3.7. Let $C$ be a convex body in $\mathbb{R}^{n}$, let $S \subseteq \mathbb{R}^{n}$, and let $0<r<1$ be arbitrary. If $S$ is $C$-ball convex, then $r S$ is $C$-ball convex. In particular, $r C$ is $C$-ball convex. Similarly, if $S$ is $C$-spindle convex, then $r S$ is $C$-spindle convex. In particular, $r C$ is $C$-spindle convex.

Proof. We apply Theorem 2 to $r C$ and $(1-r) C$.

For $n \geq 3$, the analogous implication of Theorem 2 is not true for $S+D$ in general, so ball convexity is not preserved in general by adding a convex body $D$ to both a $C$-ball convex set $S$ and to $C$. The same holds for spindle convexity. We show both in the following example.

Example 3.8. We describe convex bodies $C, D \subset \mathbb{R}^{n}$ and a set $S \subset \mathbb{R}^{n}$, for any $n \geq 3$, such that $S$ is $C$-ball convex (and thus $S$ is $C$-spindle convex), and $S+D$ is not $(C+D)$-spindle convex. We note that all sets $C, D, S \subseteq \mathbb{R}^{n}$ will be centrally symmetric. 
Let $C=\operatorname{conv}\left([-1,1]^{n} \cup\left\{ \pm(1+\varepsilon) e_{i}\right\}_{i=1}^{n-1}\right)$, where $0<\varepsilon<1$. Let $D=r B^{n}$, that is $D$ is a Euclidean ball of radius $r$ for some $r>0$, centered at the origin. Choose $S=\left[e_{n},-e_{n}\right]$. Then $S=\operatorname{conv}_{C}^{b}(S)=\operatorname{conv}_{C}^{s}(S)$ since $S=\left(C+\sum_{i=1}^{n-1} e_{i}\right) \cap\left(C-\sum_{i=1}^{n-1} e_{i}\right)$.

On the other hand, $(S+D) \cap\left\{x_{n}=0\right\}=r B^{n-1} \times\{0\}$, and thus $\pm r e_{i} \in$ $\operatorname{bd}(S+D)$ for any $1 \leq i \leq n-1$, while we have

$\pm r e_{i} \in \operatorname{int}\left(\operatorname{conv}_{C+D}^{s}\left((1+r) e_{n} \cup\left(-(1+r) e_{n}\right)\right)\right) \subseteq \operatorname{int}\left(\operatorname{conv}_{C+D}^{s}(S+D)\right)$

for any $1 \leq i \leq n-1$, so $(S+D) \subsetneq \operatorname{conv}_{C+D}^{s}(S+D)$.

In the following, we show that Example 3.1 is not a 'rare phenomenon'.

Theorem 3. Let $n \geq 3$. The family of those smooth $n$-dimensional convex bodies for which the associated ball and spindle convexity do not coincide, forms an everywhere dense set in the metric space of the $n$-dimensional convex bodies, equipped with the Hausdorff metric.

Proof. Let $C$ be any convex body. Note that there are two distinct points $x, y$ of $C$ with a hyperplane $F$ through the origin such that the parallel hyperplanes $F+x$ and $F+y$ support $C$ and their intersection with $C$ is $\{x\}$ and $\{y\}$, respectively.

Let $T$ be a small $(n-1)$-dimensional regular simplex in $F$ with the origin as its centroid. Let $C^{\prime}=\operatorname{conv}(C \cup(T+x) \cup(-T+y))$ and let $H=(T-T) / 2$. Similarly to Example 3.1, we observe that $H$ is an $(n-1)$-dimensional $C^{\prime}$ spindle convex set, whereas $\operatorname{conv}_{C^{\prime}}^{b}(H)$ is $n$-dimensional.

To construct a smooth body with respect to which ball and spindle convexity do not coincide, we take $H$ and $\bar{C}=C^{\prime}+\rho B^{n}$. If $\rho>0$ is sufficiently small, then $\bar{C}$ is close to $C$.

Now we present an example of $C$ and a closed convex set $H$ where $H$ satisfies Property (p), i.e. it is $C$-ball convex, but does not satisfy (h).

Example 3.9. Let $T$ be the Euclidean unit disk centered at the origin in the plane $U=\left\{x_{3}=0\right\}$ in $\mathbb{R}^{3}$, and consider the points $p=e_{1}$ and $q=-e_{2}$. Let $T^{\prime} \subset T+e_{3}$ be a smooth plane convex body in the $U+e_{3}$ plane with the following properties: $T^{\prime}$ is symmetric about $e_{3}$, and the chord $\left[p^{\prime}, q^{\prime}\right]$ of $T^{\prime}$ connecting its supporting lines parallel to the $x_{2}$-axis is parallel to and slightly longer than $[p, q]$. We choose our notation in a way that $T^{\prime}+p-p^{\prime}$ and $T$ are 


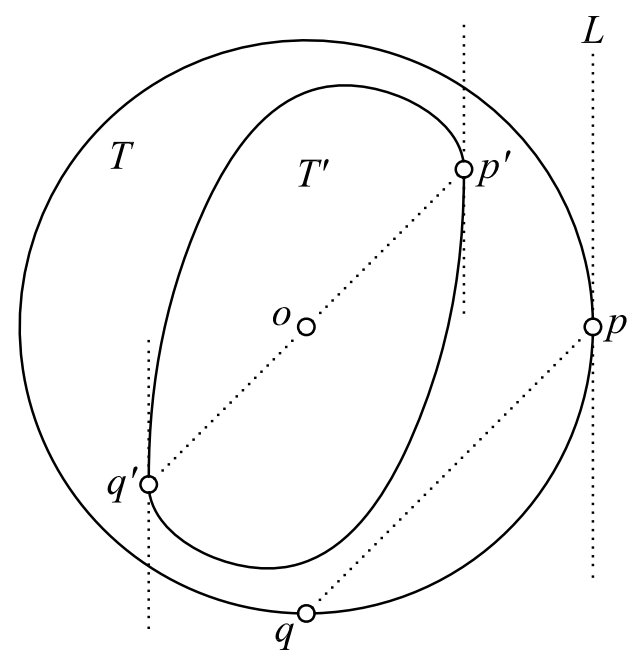

Figure 2: Example 3.9

on the same side of the line $L$ defined by the equations $x_{1}=1, x_{3}=0$. Let $p^{*}$ be the reflection of $p^{\prime}$ about the plane $U$, and set $C=\operatorname{conv}\left(T \cup T^{\prime} \cup\left(-T^{\prime}\right)\right)$. Figure 2 shows the $x_{3}=0$ and $x_{3}=1$ sections of $C$, viewing it from the top. Let $H=\left(C+p-p^{\prime}\right) \cap\left(C+p-p^{*}\right)$ and observe that $H=T^{\prime}+p-p^{\prime}$ is a $C$-ball convex set contained in $U$. Consider the plane with the equation $x_{1}=1$, and note that it supports $H$ at $p$.

If a translate $C+v$ of $C$ separates $H$ from the plane $x_{1}=1$ then $p \in C+v$, and $x_{1}=1$ is a support plane of $C+v$. It follows that $v=0$. However, $C \nsupseteq H$ because $\left|p^{\prime}-q^{\prime}\right|>|p-q|$.

A suitable modification of this example yields a smooth convex body $C$ arbitrarily close to the Euclidean unit ball such that some $C$-ball convex sets (which thus satisfy (p)) do not satisfy (h). Hence, by Remark 3.5, there are spindle convex sets that satisfy (p) and do not satisfy (s) for some convex body $C$.

We propose the following problems.

Problem 3.10. Is there a spindle convex set $K$ that satisfies ( $h$ ) and does not satisfy (s) with respect to some convex body $C$ ?

Problem 3.11. Is there a convex body $C$ such that every $C$-spindle convex set $K$ satisfies (p) (respectively (s)), but at least one of them does not satisfy (s) (respectively $(h))$ ? 
We conclude this section by finding some special classes of convex bodies such that any spindle convex set with respect to any of them satisfies Property (h). Thus, if a set is closed and spindle convex with respect to any of them, then it is ball convex with respect to the same convex body. First, we recall a standard definition.

Definition 3.12. The central symmetral $\frac{1}{2}(C-C)$ of a convex body $C \subset \mathbb{R}^{n}$ defines a norm on $\mathbb{R}^{n}$, called the relative norm of $C$ (cf. [9]). We recall that in this norm, $C$ is a convex body of constant width two. For points $p, q \in \mathbb{R}^{n}$, we call the distance between $p$ and $q$, measured in the norm relative to $C$, the $C$-distance of $p$ and $q$ (cf. Lassak [22]). For a set $X$, we denote the diameter of $X$ measured in $C$-distance by $\operatorname{diam}_{C} X$. If $C$ is the Euclidean ball, we may write simply diam $X$.

Theorem 4. Let $C \subset \mathbb{R}^{2}$ be a plane convex body. Then any $K \subset \mathbb{R}^{n} C$ spindle convex set satisfies (h).

Proof. Clearly, we may assume that $K$ is closed. We show the assertion for the case that $K$ is a plane convex body. We leave it to the reader to verify it in the cases that $K$ is not bounded or has an empty interior.

Let $K$ be a $C$-spindle convex body, and $L$ be any line supporting $K$. Since translating $C$ does not change whether $K$ satisfies (h) or not, we may assume that $L$ supports also $C$. Let $L^{\prime}$ be the other supporting line of $C$ parallel to $L$.

First, consider the case that $C \cap L$ is a singleton $\{x\}$. Note that as $K$ is $C$-spindle convex, we have that $K \cap L$ is also a singleton, since otherwise the $C$-spindle of the endpoints of $K \cap L$ has a point on the side of $L$ not containing $K$. Without loss of generality, we may assume that $K \cap L=\{x\}$.

Assume that $K \nsubseteq C$, and consider a point $y \in K \backslash C$. Observe that there is a translate of $C$ containing $x$ and $y$ if, and only if their $C$-distance is at most two. Thus, $y$ is contained in the closed unbounded strip between $L$ and $L^{\prime}$. Note that if we sweep through $C$ by a family of parallel lines, the $C$-length of the intersecting segment strictly increases while its length reaches two, then it may stay two for a while, then it strictly decreases until it reaches the other supporting line of $C$. Consider the chords of $C$ that are parallel to and not shorter than $[x, y]$, and observe that, since $y \notin C$, they all are on the side of the line, passing through $x$ and $y$, that contains $L^{\prime} \cap C$ (cf. Figure 3). From this, it follows that the intersection of all the translates of $C$ containing $x$ and $y$ (or in other words, the $C$-spindle of $x$ and $y$ ) has a 
point on the side of $L$ not containing $C$. Since $K$ is $C$-spindle convex and $L$ supports $K$ at $x$, we arrived at a contradiction.

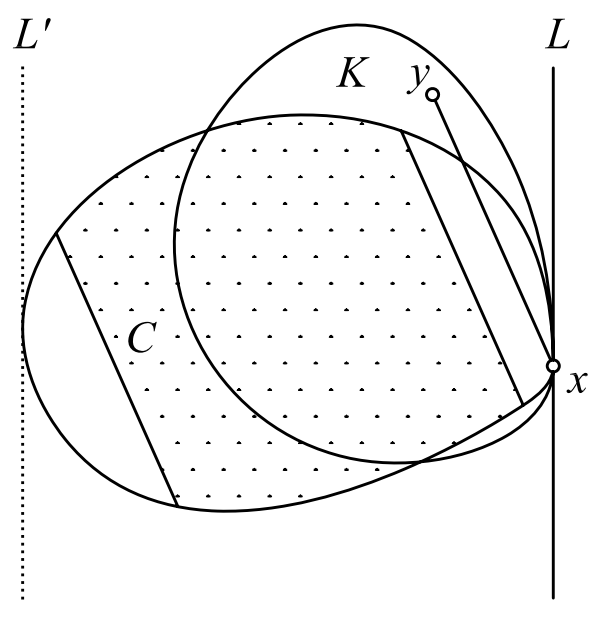

Figure 3:When $C \cap L=\{x\}$

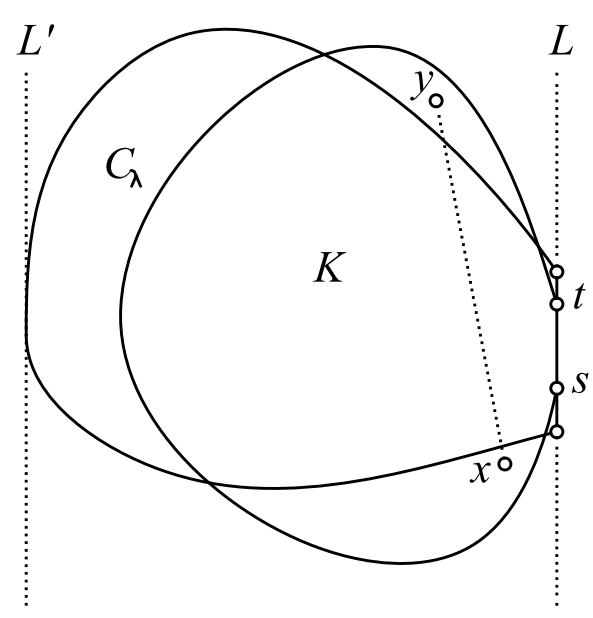

Figure 4:When $C \cap L=[p, q]$

Now consider the case that $C \cap L=[p, q]$ with $p \neq q$. Let $K \cap L=[s, t]$. Then $|s-t| \leq|p-q|$, and we may assume that $[s, t] \subset[p, q]$ and that $t \in[s, q]$. If there is a point $v \in K$ not contained in the closed strip bounded by $L$ and $L^{\prime}$, then the $C$-distance of $v$ and $s$ is greater than two; a contradiction. Set $C_{0}=t-q+C, C_{1}=s-p+C$ and $u=q-p-(s-t)$, which yields that $C_{1}=u+C_{0}$.

Assume that there is no translate of $C$, with supporting line $L$, that contains $K$. Then, $C_{\lambda} \backslash K \neq \emptyset$ for every $\lambda \in[0,1]$, where $C_{\lambda}=\lambda u+C_{0}$. Let $H$ denote the closed strip bounded by $L$ and $L^{\prime}$. For simplicity, we regard the connected component of $H \backslash C_{\lambda}$, not containing $q$ as the one below $C_{\lambda}$, and the other one as the one above $C_{\lambda}$. It is easy to see that if there is no $\lambda$ with $K \subset C_{\lambda}$, then there is a value of $\lambda$ such that both components of $H \backslash C_{\lambda}$ contain a point of $K$. Let $\lambda$ be such a value, and let $x, y \in K$ be points such that $x$ is in the component below $C_{\lambda}$ and $y$ is in the component above $C_{\lambda}$ (cf. Figure 4).

Consider the segment $[x, y]$ and note that if their $C$-distance is greater than two, then $[x, y]_{C}=\mathbb{R}^{2}$. Thus, all the chords of $C_{\lambda}$ that are parallel to and not shorter than $[x, y]$ are on the same side of the line containing $[x, y]$. Similarly like in the previous case, it follows that $[x, y]_{C} \not \subset H$, which contradicts our assumption that $K \nsubseteq H$. 
Corollary 3.13. For a plane convex body $C \subset \mathbb{R}^{2}$, any closed $C$-spindle convex set is $C$-ball convex.

Proposition 3.14. Let $C_{1} \subset \mathbb{R}^{k}$ and $C_{2} \subset \mathbb{R}^{m}$ be convex bodies, and consider a set $S \subset \mathbb{R}^{k} \oplus \mathbb{R}^{m}=\mathbb{R}^{k+m}$. Let proj $_{1}$ and proj $_{2}$ be the orthogonal projections of $\mathbb{R}^{k} \oplus \mathbb{R}^{m}$ on the first and second factor, respectively. Then,

$$
\begin{aligned}
& \operatorname{conv}_{C_{1} \times C_{2}}^{b}(A)=\operatorname{conv}_{C_{1}}^{b}\left(\operatorname{proj}_{1} A\right) \times \operatorname{conv}_{C_{2}}^{b}\left(\operatorname{proj}_{2} A\right) ; \\
& \operatorname{conv}_{C_{1} \times C_{2}}^{s}(A)=\operatorname{conv}_{C_{1}}^{s}\left(\operatorname{proj}_{1} A\right) \times \operatorname{conv}_{C_{2}}^{s}\left(\operatorname{proj}_{2} A\right) .
\end{aligned}
$$

Proof. Note that for any $x \in \mathbb{R}^{k+m}, x+\left(C_{1} \times C_{2}\right)=\left(\operatorname{proj}_{1} x+C_{1}\right) \times\left(\operatorname{proj}_{2} x+\right.$ $\left.C_{2}\right)$. Thus, $A \subseteq x+\left(C_{1} \times C_{2}\right)$ is equivalent to $\operatorname{proj}_{1} A \subseteq\left(\operatorname{proj}_{1} x+C_{1}\right)$ and $\operatorname{proj}_{2} A \subseteq\left(\operatorname{proj}_{2} x+C_{2}\right)$. This immediately yields the equality of the ball convex hulls. It follows that for any points $p, q \in \mathbb{R}^{k+m}$, we have $[p, q]_{C_{1} \times C_{2}}=$ $\left[\operatorname{proj}_{1} p, \operatorname{proj}_{1} q\right]_{C_{1}} \times\left[\operatorname{proj}_{2} p, \operatorname{proj}_{2} q\right]_{C_{2}}$. Hence, the right-hand side in the second equality contains the left-hand side. Now consider any $\left(C_{1} \times C_{2}\right)$ spindle convex set $K$. We show that $\operatorname{proj}_{1} K \times \operatorname{proj}_{2} K \subseteq K$. Consider points $p_{1} \in \operatorname{proj}_{1} K$ and $q_{2} \in \operatorname{proj}_{2} K$. We need to show that $\left(p_{1}, q_{2}\right) \in K$. Note that $\left(p_{1}, p_{2}\right) \in K$ and $\left(q_{1}, q_{2}\right) \in K$ for some $p_{2} \in \mathbb{R}^{m}$ and $q_{1} \in \mathbb{R}^{k}$. Thus, by applying the first equality for $\left(C_{1} \times C_{2}\right)$-spindles, we obtain that $\left(p_{1}, q_{2}\right) \in K$, which is what we wanted to prove.

Corollary 3.15. If $C$ is an $n$-dimensional axis-parallel cube, and $A \subseteq \mathbb{R}^{n}$ is any set, then both $\operatorname{conv}_{C}^{b}(A)$ and $\operatorname{conv}_{C}^{s}(A)$ are either the axis-parallel brick containing $A$ and minimal with respect to inclusion, or $\mathbb{R}^{n}$.

\section{Arc-distance defined by $C$}

In the theory of spindle convexity with respect to the Euclidean disk, there is a naturally arising associated distance function. This distance is called arc-distance, and is defined for points $p, q \in \mathbb{R}^{2}$ as the Euclidean length of a shortest unit circle arc connecting the points (cf. [3] and [5]). The aim of this section is to generalize this distance for spindle convexity with respect to any origin-symmetric plane convex body.

Let $C$ be a planar $O$-symmetric convex body, that is the unit disk of a normed plane. We recall that the $C$-length of a polygonal curve is the sum of the $C$-distances between the consecutive pairs of points, and that the $C$-length of a curve is the supremum, if it exists, of the $C$-lengths of the 
polygonal curves for which all the vertices are chosen from the curve. If $D$ is a convex body, then the perimeter of $D$ with respect to $C$ is the $C$-length of the boundary of $D$. We denote this quantity by $\operatorname{perim}_{C} D$. It is known that for any plane convex bodies $C$ and $D$, we have $\operatorname{perim}_{C} D=\operatorname{perim}_{C}\left(\frac{1}{2}(D-D)\right)$ (cf. [10]), and $6 \leq \operatorname{perim}_{C} C \leq 8$ (cf. [11] or [30]).

Definition 4.1. Let $C$ be an o-symmetric plane convex body, and let $p, q$ be points at $C$-distance at most two. Then the arc-distance $\rho_{C}(p, q)$ of $p, q$ with respect to $C$ is the minimum of the $C$-lengths of the arcs, with endpoints $p$ and $q$, that are contained in $\operatorname{bd}(y+C)$ for some $y \in \mathbb{R}^{2}$.

Definition 4.2. Let $C$ be an o-symmetric plane convex body, $z \in \mathbb{R}^{2}$ and $0 \leq \rho \leq \frac{1}{2} \operatorname{perim}_{C} C$. Then the arc-distance disk, with respect to $C$, of center $z$ and radius $\rho$ is the set

$$
\Delta_{C}(z, \rho)=\left\{w \in \mathbb{R}^{2}: \rho_{C}(z, w) \leq \rho\right\} .
$$

Furthermore, we set $\Delta_{C}(\rho)=\Delta_{C}(o, \rho)$.

Clearly, for any $C$, arc-distance disks of the same radius are translates of each other, but those of different radii are not necessarily even similar. We note that if $C$ is a Euclidean disk, then its arc-distance disks are Euclidean disks.

Theorem 5. For any o-symmetric plane convex body $C$ and $0 \leq \rho \leq$ $\frac{1}{2} \operatorname{perim}_{C} C$, the arc-distance disk $\Delta_{C}(\rho)$ is convex.

Proof. Note that by compactness arguments, it is sufficient to prove the assertion for the case that $C$ is a convex polygon with, say, $m$ vertices and for values of $\rho$ such that $0 \leq \rho \leq \frac{1}{2} \operatorname{perim}_{C} C$ is not equal to the sum of some sides of $C$. In this case no chord of $C$ connecting two vertices determines an arc of length $\rho$.

Let us move a point $p(t)$ around on bd $C$ at a constant speed measured in $C$-distance, and consider the point $q(t)$ such that $\rho_{C}(p(t), q(t))=\rho$. Note that on any side of $C$, the points $p(t)$ and $q(t)$ move at a constant speed also in the Euclidean metric, and their Euclidean speed is proportional to the lengths of the longest chords of $C$ parallel to the corresponding edges of $C$. Thus, the vector $q(t)-p(t)$ is a linear function of $t$ if $p(t)$ and $q(t)$ are on different edges, and a constant if they are on the same edge, which yields that $Q=\operatorname{bd} \Delta_{C}(\rho)$ is a (starlike) polygonal curve with at most $2 m$ vertices. 


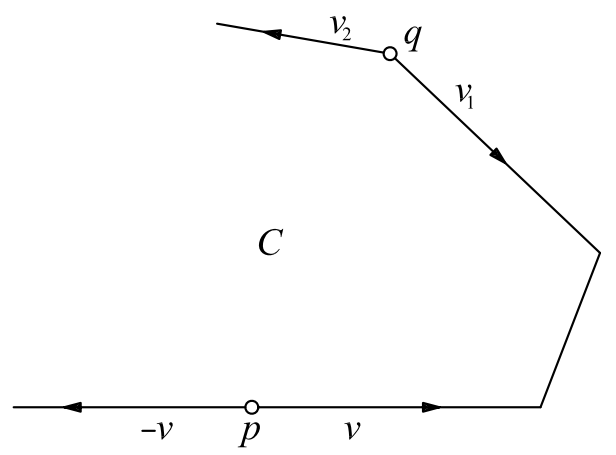

Figure 5: Polygon $C$ in the proof of Theorem 5

We show that $Q$ is convex. Since $Q$ is clearly star-like with respect to the origin, it is sufficient to prove that for each vertex of $Q$ if we consider that vertex, one point on each edge meeting at that vertex and the origin, then these four points are in convex position. Any vertex of $Q$ is of the form $q-p$, where $q$ is a vertex of $C$, and $p$ is a point in the relative interior of some edge of $C$.

Consider the sufficiently small vectors $v, v_{1}$ and $v_{2}$ whose Euclidean lengths are proportional to the Euclidean lengths of the longest chords of $C$ parallel to them, and such that $p-v, p, p+v$ are on the same edge of $C$, and $q+v_{1}$ and $q+v_{2}$ are on the two consecutive edges of $C$ meeting at $q$. We choose our notation in a way that the line passing through $[p, q]$ separates $p-v$ and $q+v_{2}$ from $p+v$ and $q+v_{1}$, and that $v,-v_{1}$ and $v_{2}$ are in counterclockwise order (cf. Figure 5). Note that as $C$ is convex, the segment $\left[q+v_{1}, q+v_{2}\right]$ intersects $[p, q]$.

Now, to show that $Q$ is convex, we need to show that the points $o, q+$ $v_{1}-(p-v), q-p$ and $q+v_{2}-(p+v)$ are in convex position. Adding $p$ to each point, we are considering the quadrilateral with vertices $p, q+v_{1}+v, q$ and $q+v_{2}-v$. It is clearly convex by the convexity of $C$.

We note that if $C$ is not $o$-symmetric, we may define a non-symmetric arc-distance, for which we may prove the assertion of Theorem 5 using a similar technique.

Remark 4.3. If $C$ is smooth, then $\Delta_{C}(\rho)$ is smooth for any positive value of $\rho$. 
Proof. Consider the $C$-arc-length parametrization $\Gamma:[0, \alpha] \rightarrow \mathbb{R}^{2}$ of bd $C$. Then bd $\Delta_{C}(\rho)$ is the graph of the curve $\tau \mapsto \Gamma(\tau+\rho)-\Gamma(\tau)$. If $\Gamma$ is differentiable, then so is this function.

Our next corollary follows from Theorem 5 and the fact that $\Delta_{C}\left(\rho_{1}\right) \subset$ $\Delta_{C}\left(\rho_{2}\right)$ for any $\rho_{1}<\rho_{2}$.

Corollary 4.4. For any $x, y, z \in \mathbb{R}^{2}$ and an o-symmetric plane convex body $C$, the function $\tau \mapsto \rho_{C}(x, y+\tau z)$ is quasiconvex on its domain; that is: it consists of a strictly decreasing, possibly a constant and then a strictly increasing interval.

We prove the following version of the triangle inequality for arc-distance, which, for the Euclidean case, appeared first as Lemma 1 in [3].

Theorem 6. Let $C$ be an o-symmetric plane convex body, and let $x, y, z \in \mathbb{R}^{2}$ be points such that each pair has a $C$-arc-distance.

(1) If $y \in \operatorname{int}[x, z]_{C}$, then $\rho_{C}(x, y)+\rho_{C}(y, z) \leq \rho_{C}(x, z)$,

(2) if $y \in \mathrm{bd}[x, z]_{C}$, then $\rho_{C}(x, y)+\rho_{C}(y, z)=\rho_{C}(x, z)$, and

(3) if $y \notin[x, z]_{C}$ and $C$ is smooth, then $\rho_{C}(x, y)+\rho_{C}(y, z) \geq \rho_{C}(x, z)$.

Proof. The assertion of (2) is trivial. Assume that $y \in \operatorname{int}[x, z]_{C}$. Clearly, there is a line $L$ containing $y$ such that $L \cap \mathrm{bd}[x, z]_{C}=\{a, b\}$ with $\rho_{C}(x, a)=$ $\rho_{C}(x, b)$ and $\rho_{C}(z, a)=\rho_{C}(z, b)$. Thus, the assertion in this case is a consequence of Corollary 4.4 .

Assume that $C$ is smooth and $y \notin[x, z]_{C}$. If $\rho_{C}(x, y) \geq \rho_{C}(x, z)$, then there is nothing to prove, and thus, we may assume that $y \in \operatorname{int} \Delta_{C}\left(x, \rho_{C}(x, z)\right)$. Similarly, we may assume that $y \in \operatorname{int} \Delta_{C}\left(z, \rho_{C}(x, z)\right)$. For any $0<\tau<$ $\rho_{C}(x, z)$, let $L(\tau)$ denote the line for which $L(\tau) \cap \mathrm{bd}[x, z]_{C}=\{c, d\}$ with $\rho_{C}(x, c)=\rho_{C}(x, d)=\tau$, and let $R$ be the region swept through by the lines $L(\tau), 0<\tau<\rho_{C}(x, z)$. Since $C$ is smooth, the limit $L_{+}$of $L(\tau)$ as $\tau$ approaches $\rho(x, z)$ is the supporting line of $\Delta_{C}(x, \rho(x, z))$ at $z$. Similarly, if $\tau \rightarrow 0$, the limit $L_{-}$of $L(\tau)$ is the supporting line of $\Delta_{C}\left(z, \rho_{C}(x, z)\right)$ at $x$. Since $\Delta_{C}\left(x, \rho_{C}(x, z)\right) \cap \Delta_{C}\left(z, \rho_{C}(x, z)\right)$ lies between the parallel lines $L_{+}$and $L_{-}$, and this open unbounded strip is clearly contained in $R, y \in L(\tau)$ for some value of $\tau$, and (3) follows from Corollary 4.4.

Example 4.5. Let $C$ be the unit ball of the $l_{\infty}$ norm in $\mathbb{R}^{2}$. Then

$$
\Delta(\rho)= \begin{cases}\left\{\left(x_{1}, x_{2}\right) \in \mathbb{R}^{2}:\left|x_{1}\right|+\left|x_{2}\right| \leq \rho\right\}, & \text { if } 0<\rho \leq 2 \\ \left\{\left(x_{1}, x_{2}\right) \in \mathbb{R}^{2}:\left|x_{1}\right|+\left|x_{2}\right| \leq \rho,|x| \leq 2,|y| \leq 2\right\}, & \text { if } 2<\rho \leq 4\end{cases}
$$


In this example, for any two points $x, z$ with $\rho_{C}(x, z) \leq 2$, we have $\rho_{C}(x, y)+\rho_{C}(y, z)=\rho(x, z)$ for any $y \in[x, z]_{C}$. Furthermore, if we replace the corners of $C$ with small circle arcs, then the boundary of the arc-distance balls of the obtained body consists 'almost only' of segments that are parallel to the segments in bd $\Delta(\rho)$. Thus, we may create a smooth convex body and points $x, y, z$ with $y \notin \operatorname{int}[x, z]_{C}$ such that $\rho_{C}(x, y)+\rho_{C}(y, z)=\rho_{C}(x, z)$.

We propose the following questions.

Problem 4.6. Can we drop the smoothness condition in part (3) of Theorem 6 ?

Problem 4.7. Prove or disprove that if $C$ is strictly convex, then $\Delta_{C}(\rho)$ is strictly convex for any $0<\rho \leq \frac{1}{2} \operatorname{perim}_{C} C$.

Problem 4.8. Prove or disprove that if $C$ is strictly convex, then the inequalities in (1) and (3) of Theorem 6 are strict.

\section{Carathéodory numbers}

Now we recall the notion of the Carathéodory-number of a convexity space (cf. [21], [29] and [33]).

Definition 5.1. Let $(X, \mathcal{G})$ be a convexity space (for the definition, see Section 2). The Carathéodory number $\operatorname{Car} \mathcal{G}$ of $\mathcal{G}$ is the smallest positive integer $k$ such that for any $V \subseteq X$ and $p \in \operatorname{conv}_{\mathcal{G}}(V)$ there is a set $W \subseteq V$ with card $W \leq k$ and with $p \in \operatorname{conv}_{\mathcal{G}}(W)$. If no such positive integer exists, we set $\operatorname{Car} \mathcal{G}=\infty$.

Definition 5.2. Let $C \subset \mathbb{R}^{n}$ be a convex body, and let $\mathcal{G}_{1}$ (respectively $\mathcal{G}_{2}$ ) be the family of closed $C$-spindle convex sets (respectively, the $C$-ball convex sets) in $\mathbb{R}^{n}$. Then we call Car $\mathcal{G}_{1}$ (respectively Car $\mathcal{G}_{2}$ ) the spindle Carathéodory number (respectively, ball Carathéodory number) of $C$, and denote it by $\mathrm{Car}^{s} \mathrm{C}$ (respectively, by $\mathrm{Car}^{b} \mathrm{C}$ ).

These numbers were determined in [5] for the Euclidean ball $B^{n}$ as $C$.

Theorem 7. Let $C \subset \mathbb{R}^{2}$ be a plane convex body. If $C$ is a parallelogram, then both Carathéodory numbers of $C$ are two, otherwise both are three. 
Proof. By Theorem 4, for any $C \subset \mathbb{R}^{2}$, a closed set is $C$-spindle convex if, and only if it is $C$-ball convex. Thus, the two Carathéodory numbers of $C$ are equal.

Let $X \subset \mathbb{R}^{2}$ be any closed set. The $C$-ball convex hull of $X$ is the intersection of all the translates of $C$ that contain $X$. If $X$ is a singleton, the assertion immediately follows, and thus, we assume that card $X>1$. If no translate of $C$ contains $X$, then, by Helly's theorem, there are at most three points of $X$ that are not contained in any translate of $C$.

Assume that there is a translate of $C$ containing $X$. Consider a point $p \in \mathrm{bd} \mathrm{conv}_{C}^{s}(X)$. If $p \in X$, we are done. Assume that $p \notin X$. We leave it to the reader to show that there is a translate $u+C$ with the property that $\operatorname{bd}(u+C)$ contains $p$ and two distinct points $z_{1}, z_{2} \in X$, such that the $C$-distance of $z_{1}$ and $z_{2}$ is 2 , or, if the $C$-distance of $z_{1}$ and $z_{2}$ is less than two, then the connected component of $(u+C) \backslash\left[z_{1}, z_{2}\right]$ containing $p$ does not contain points at $C$-distance two. Without loss of generality, we may assume that the open arc in $\operatorname{bd}(u+C)$ with endpoints $z_{1}$ and $z_{2}$ that contains $p$ is disjoint from $X$.

If the $C$-distance of $z_{1}$ and $z_{2}$ is less than two, then, clearly, $p$ is contained in any translate of $C$ that contains $z_{1}$ and $z_{2}$, or, in other words, $p \in\left[z_{1}, z_{2}\right]_{C}$. If the $C$-distance of $z_{1}$ and $z_{2}$ is equal to two, there are two parallel lines $L_{1}$ and $L_{2}$ that support $u+C$ at $z_{1}$ and $z_{2}$, respectively. Since $y \in \operatorname{conv}_{C}^{s}(X)$, we have for $i=1,2$ that $z_{i}$ is the endpoint of the segment $L_{i} \cap(u+C)$ closer to $p$. Thus, $p \in\left[z_{1}, z_{2}\right]_{C}$.

Now consider a point $p \in \operatorname{int} \operatorname{conv}_{C}^{s}(X)$. Let $v \in X$ be arbitrary. Choose a point $z \in \operatorname{bdconv}_{C}^{s}(X)$ such that $p \in[v, z]$. Then, by the previous paragraph, there are points $z_{1}, z_{2} \in X$ such that $z \in\left[z_{1}, z_{2}\right]_{C}$, and clearly, $p \in \operatorname{conv}_{C}^{s}\left(\left\{v, z_{1}, z_{2}\right\}\right)$.

Finally, assume that $C$ is not a parallelogram. Note that in that case there are three smooth points in bd $C$ such that the unique lines supporting $C$ at them are the sidelines of a triangle containing $C$. Let $X$ be the vertex set of this triangle. Observe that for a sufficiently large $\lambda>0$, the centroid $c$ of $X$ is not contained in the $(\lambda C)$-spindles determined by any two points of $X$. Since $c \in \operatorname{conv}(X) \subseteq \operatorname{conv}_{\lambda C}^{s}(X)$ for any value of $\lambda$, we have that both Carathéodory numbers of $C$ are three. The observation that they are two if $C$ is a parallelogram follows from the next theorem.

Proposition 5.3. If $C$ is an $n$-dimensional parallelotope, then $\operatorname{Car}^{s} C=$ $\operatorname{Car}^{b} C=n$. 
Proof. Since both Carathéodory numbers are affine invariant quantities, we may assume that $C$ is the unit ball of the $l_{\infty}$ norm. By Corollary 3.15, the two Carathéodory numbers of $C$ are equal. Let $X$ be a closed set in $\mathbb{R}^{n}$. Then $\operatorname{conv}_{C}^{s}(X)$ is either $\mathbb{R}^{n}$ or the minimal volume axis-parallel brick that contains $X$. In the first case it is easy to see that there are two points of $X$ that are not contained in any translate of $C$, from which the assertion readily follows.

Assume that $\operatorname{conv}_{C}^{s}(X)$ is the minimal volume axis-parallel brick that contains $X$, and consider a point $y \in \operatorname{conv}_{C}^{s}(X)$. Then, by a theorem of Lay [24], there is a subset $X^{\prime} \subseteq X$ with card $X^{\prime} \leq n$ such that the minimal volume axis-parallel brick containing $X^{\prime}$ contains $y$. Thus, we obtain $y \in \operatorname{conv}_{C}^{s}\left(X^{\prime}\right)$ and hence $\operatorname{Car}^{s} C \leq n$.

On the other hand, let $X$ be the $n$ neighbors of a given vertex $v$ of $C$. Then $\operatorname{conv}_{C}^{s}(X)=C$. Let $y$ be the vertex of $C$ opposite of $v$, and observe that, removing any point of $X$, the $C$-ball convex hull of the remaining points is a facet of $C$ not containing $y$. Thus, $\operatorname{Car}^{s} C \geq n$.

Similarly to Corollary 3.15 and Proposition 5.3, we can prove the following.

Proposition 5.4. Let $C \subset \mathbb{R}^{n}$ be a simplex.

(i) For any closed set $X \subset \mathbb{R}^{n}$, both $\operatorname{conv}_{C}^{b}(X)$ and $\operatorname{conv}_{C}^{s}(X)$ are either $\mathbb{R}^{n}$, or the smallest positive homothetic copy of $C$ that contains $C$.

(ii) $\operatorname{Car}^{s} C=\operatorname{Car}^{b} C=n+1$.

We apply the following remark several times in this section.

Remark 5.5. For any set $H \subset \mathbb{R}^{n}$, a point $p \in \mathbb{R}^{n}$, and a convex body $C \subset \mathbb{R}^{n}$, we have that $p \in \mathbf{B}_{C}^{-}(H)$ if, and only if, $C+p \supseteq H$, and similarly, $p \in \mathbf{B}_{C}^{+}(H)$ if, and only if, $-C+p \supseteq H$.

Proposition 5.6. Let $C$ be a polytope in $\mathbb{R}^{n}$ with $k$ facets. Then the ball Carathéodory number of $C$ is at most $k n$.

Proof. Let $X \subset \mathbb{R}^{n}$ and $p \in \operatorname{conv}_{C}^{b}(X)$ be given. We need to find a subset $Y$ of $X$ of cardinality $n k$ such that $p \in \operatorname{conv}_{C}^{b}(Y)$. By Remark 5.5, we have that $C \supseteq \mathbf{B}_{C}^{-}(X)$, that is, $\left(\mathbb{R}^{n} \backslash C\right) \cap \bigcap_{v \in X}(-C+v)=\emptyset$. Since $\mathbb{R}^{n} \backslash C$ is the union of $k$ convex sets, the statement follows from Helly's theorem. 
The following construction, similar to Example 4 in [27], shows that in $\mathbb{R}^{3}$ there are convex bodies with arbitrarily large ball Carathéodory numbers.

Theorem 8. Let $k \in \mathbb{Z}^{+}$be given. Then there is an o-symmetric convex body $C \subset \mathbb{R}^{3}$ and a set $X \subset \mathbb{R}^{3}$ such that $o \in \operatorname{conv}_{C}^{b}(X)$, but for any $Y \subset X$, $|Y|<k$ we have that $o \notin \operatorname{conv}_{C}^{b}(Y)$.

Proof. We may assume that $k$ is even. Consider the paraboloid $P$ in $\mathbb{R}^{3}$ defined by $x_{3}=x_{1}^{2}+x_{2}^{2}$. We choose $k$ points on the parabola $P_{0}=P \cap\left\{x_{1}=\right.$ $0\}$, and number these points according to the order in which they lie on the parabola: $U=\left\{u_{1}, u_{2}, \ldots, u_{k}\right\} \subset P_{0}$. Plane sections of $P$ parallel to the $x_{2} x_{3}$-plane are translates of this parabola. Let $P_{i}=P \cap\left\{x_{1}=i\right\}$ for $i \in I$, where $I=\left\{-\frac{k}{2},-\frac{k}{2}+1, \ldots,-2,-1,1,2, \ldots, \frac{k}{2}\right\}$. Now, for each $i \in I$, there is a unique translation vector $t_{i}$ such that $P_{0}=P_{i}+t_{i}$. Let $U_{i}=\left(U \backslash\left\{u_{i}\right\}\right)-t_{i} \subset P_{i}$. Let $h>0$ be larger than the largest $x_{3}$ coordinate of the points in any $U_{i}$. Finally, consider the (bounded) arc of $P_{0}$ that lies in the half-space $\left\{x_{3} \leq h\right\}$. Delete from this arc very small open arcs around each point of $U$, and call the remaining part of $P_{0}$ (the union of $k+1$ closed bounded arcs) $U_{0}$. We define $C$ as the following $O$-symmetric convex body:

$$
C=\operatorname{conv}\left[\left(\bigcup_{i=0}^{k} U_{i}-(0,0, h)\right) \bigcup-\left(\bigcup_{i=0}^{k} U_{i}-(0,0, h)\right)\right] .
$$

Let $X=\left\{t_{i}: i \in I\right\}$. Now, $\mathbf{B}_{C}(X) \subseteq \mathbf{B}_{C}\left\{t_{-\frac{k}{2}}, t_{\frac{k}{2}}\right\}$, and the latter is contained in the $x_{1}=0$ plane. Moreover, $\mathbf{B}_{C}(X)$ is contained in the planar region $\operatorname{conv}\left(P_{0}-(0,0, h)\right) \cap-\operatorname{conv}\left(P_{0}-(0,0, h)\right)$. If the open arcs in the definition of $U_{0}$ are sufficiently small then a little more is true: $\mathbf{B}_{C}(X)$ is contained in the planar region $\operatorname{conv}\left(U_{0}-(0,0, h)\right) \cap-\operatorname{conv}\left(U_{0}-(0,0, h)\right)$. Thus, $\mathbf{B}_{C}(X) \subset C$. It follows, by Remark 5.5, that $o \in \mathbf{B}_{C} \mathbf{B}_{C}(X)=$ $\operatorname{conv}_{C}^{b}(X)$. On the other hand, for any $i \in I$, we have that $u_{i} \in \mathbf{B}_{C}\left(X \backslash\left\{t_{i}\right\}\right)$, and hence, $C \not \supset \mathbf{B}_{C}\left(X \backslash\left\{t_{i}\right\}\right)$. It follows (again by Remark 5.5) that $o \notin$ $\operatorname{conv}_{C}^{b}\left(X \backslash\left\{t_{i}\right\}\right)$.

This example may be modified in several ways. First, we may generalize it for $\mathbb{R}^{n}$ with $n>3$, by replacing $C$ with $C \times[-1,1]^{n-3}$ and leaving $X$ unchanged. Second, by "smoothening" $C$, we may obtain a smooth and strictly convex $O$-symmetric body $C$ in $\mathbb{R}^{n}$ with an arbitrarily large ball Carathéodory number. Third, we may replace $U_{0}$ by a sufficiently dense finite subset of $U_{0}$, 
and thus, obtain a polytope as $C$. Finally, the following modification of the example yields an $o$-symmetric convex body in $\mathbb{R}^{3}$ whose ball Carathéodory number is infinity: In the construction, replace the finitely many $P_{i}$ s with planar sections of $P$ of the form $P_{i}=P \cap\left\{x_{1}=a_{i}\right\}$ where $a_{1}, a_{2}, \ldots$ is a sequence of real numbers in $(-1,1)$, which is symmetric about zero and which does not contain any of its accumulation points. Then, construct $U$ similarly: let $u_{1}, u_{2}, \ldots$ be a bounded sequence of points on $P_{0}$, which does not contain any of its accumulation points.

Problem 5.7. For $n \geq 3$, find the minima of the ball/spindle Carathéodory numbers of the $n$-dimensional convex bodies, and if it exists, find the maximum of their spindle Carathéodory numbers.

Problem 5.8. For $n \geq 3$, prove or disprove the existence of a convex body $C \subset \mathbb{R}^{n}$ such that its two Carathéodory numbers are different.

Problem 5.9. It is known (cf. [5]) that both Carathéodory numbers of the $n$-dimensional Euclidean ball are $n+1$. Prove or disprove that it holds also in a small neighborhood of the Euclidean ball. If the answer is negative, is the set of ball (resp., spindle) Caratheodory numbers bounded from above in a neighborhood of the Euclidean ball?

\section{When every $C$-ball convex set is finitely generated}

Clearly, every $C$-spindle is $C$-ball convex, but the converse is not true in general. However, there are convex bodies $C$ for which every $C$-ball convex set is a $C$-spindle, such as the simplices and rectangular boxes of $\mathbb{R}^{n}$, see Corollary 3.15 and Proposition 5.4. In this section, we examine a more general problem: We investigate those convex bodies $C$ that have the property that every $C$-ball convex set is the $C$-ball convex hull of finitely many points.

Definition 6.1. If every $C$-ball convex set is the $C$-ball convex hull of at most $k$ points, for some fixed $k \geq 2$, then we say that every $C$-ball convex set is $k$-generated. Similarly, if every $C$-ball convex set is the $C$-ball convex hull of finitely many points, then we say that every $C$-ball convex set is finitely generated.

By an $n$-polytope we mean an $n$-dimensional polytope.

Theorem 9. If $C$ is a convex body in $\mathbb{R}^{n}$ for which every $C$-ball convex set is k-generated, then $C$ is an n-polytope with at most $k n$ facets. 
This theorem is a consequence of the next three lemmas. The first of these readily follows by the minimality property of $C$-ball convex hull as the intersection of translates of $C$.

Lemma 6.2. Let $C$ be a convex body in $\mathbb{R}^{n}$. Let $K \subseteq \mathbb{R}^{n}$ be a bounded set, and assume that $K=\operatorname{conv}_{C}^{b}(S)$ for some closed set $S \subseteq K$. Then, a translate $C+v$ contains $K$ and fulfils $\operatorname{bd}(C+v) \cap K \neq \emptyset$ if and only if $\operatorname{bd}(C+v) \cap S \neq \emptyset$.

Lemma 6.3. If $C$ is a convex polytope in $\mathbb{R}^{n}$ for which every $C$-ball convex set is $k$-generated, then $C$ has at most $k n$ facets.

Lemma 6.4. If $C$ is a convex body in $\mathbb{R}^{n}$ for which every $C$-ball convex set is finitely generated, then $C$ is an n-polytope.

Proof of Lemma 6.3. We can obtain $\frac{1}{2} C$ as the intersection of finitely many translates of $C: \frac{1}{2} C=\bigcap_{i=1}^{m}\left(C+v_{i}\right)$. We perturb the translation vectors $v_{i}$ to obtain another polytope $P=\bigcap_{i=1}^{m}\left(C+w_{i}\right)$ in a way that $\left|v_{i}-w_{i}\right|<\varepsilon$ for a sufficiently small $\varepsilon>0$, and $P$ is a simple polytope (that is, every vertex of $C$ is contained in exactly $n$ facets of $C$ ), and each facet of $P$ is contained in the relative interior of a facet of $C+w_{i}$ for some value of $i$, and the $n_{i} \mathrm{~s}$ are pairwise distinct. By Lemma 6.2, there are $k$ points of $P$ such that every facet of $P$ contains at least one of these points. Since any point is contained in at most $n$ facets of $P$, we obtain $m \leq k n$.

Proof of Lemma 6.4. Assume that $C$ is not an $n$-polytope, and let $0<r<1$. It follows from Theorems 2.2.4 and 2.2.9 of [31] that there is an infinite sequence of pairwise distinct triplets $T_{i}=\left(p_{i}, H_{i}, n_{i}\right), i=1,2,3, \ldots$, such that $p_{i}$ is a smooth boundary point of $r C, H_{i}$ is the unique supporting hyperplane of $r C$ at $p_{i}$, and $n_{i}$ is the outer normal unit vector of $H_{i}$ with respect to $r C$, for which $n_{i} \neq n_{j}$ if $i \neq j$. To see this directly, it is also easy to construct such triplets applying induction.

We choose an infinite subsequence $\left\{T_{i} \mid i \in I\right\}$ of the triplets, for which there is at most one cluster point of $\left\{p_{i} \mid i \in I\right\}$ and $\left\{n_{i} \mid i \in I\right\}$, resp., and that cluster point is not equal to any $p_{i}$ and $n_{i}$.

Let $H_{i}^{+}$be the half-space determined by $H_{i}$ which contains $r C$. Let $r<r^{\prime}<1$. Let $C_{i}$ be that translate of $r^{\prime} C$ which touches both $r C$ and $H_{i}$ at $p_{i}, i \in I$. Let $P=\bigcap_{i \in I} C_{i}$. Since there is no cluster point among the points $p_{i}$ and vectors $n_{i}(i \in I)$, we can define a sequence $B_{i}$ of balls of positive radii such that for every $i \in I, B_{i}$ is centered at $p_{i}$, and $B_{i} \subseteq \operatorname{int}\left(C_{j}\right)$ for any $j \neq i$. 
Therefore, every $C_{i}$ can be translated by a vector $v_{i}$ towards the direction $n_{i}$ within a sufficiently small, but positive distance such that the translates $C_{i}^{\prime}=C_{i}+v_{i}, i \in I$ form $P^{\prime}=\bigcap_{i \in I} C_{i}^{\prime}$ in such a way that $H_{i}^{\prime}=H_{i}+v_{i}$ is the unique supporting hyperplane of $P^{\prime}$ at $p_{i}^{\prime}=p_{i}+v_{i}$, and at most $n$ hyperplanes have a common point among the hyperplanes $H_{i}(i \in I)$.

Then, since the smaller homothetic copies $C_{i}$ and $C_{i}^{\prime}(i \geq 1)$ of $C$ are $C$-ball convex sets by Corollary 3.7, by assumption, $P^{\prime}$ is a $C$-ball convex hull of finitely many points, so there should be a finite subset of $\operatorname{bd}\left(P^{\prime}\right)$ such that every face $H_{i}^{\prime} \cap P_{i}^{\prime}$ contains at least one element of $S$ (otherwise there would be a translate of $C$ touching $P^{\prime}$ and having a disjoint boundary from those points, and by Lemma 6.2, that would contradict to the fact that $P^{\prime}$ is a $C$-ball convex hull of those points). But one point can be contained in at most $n$ faces among the infinitely many faces $H_{i}^{\prime} \cap P_{i}^{\prime}$, so $P^{\prime}$ can not be the $C$-ball convex hull of finitely many points.

Theorem 9 implies the following corollary.

Corollary 6.5. Let $C$ be a convex body in $\mathbb{R}^{n}$. If every $C$-ball convex set in $\mathbb{R}^{n}$ is a $C$-spindle, then $C$ is an n-polytope and it has at most $2 n$ facets.

While Corollary 6.5 is sharp for parallelotopes, we do not know if it is the case for Theorem 9 for $k \geq 3$. We ask the following question.

Problem 6.6. Let $k \geq 3$ be arbitrary. Is there a convex n-polytope $C$ having $k n$ facets such that every $C$-ball convex set is $k$-generated?

In the first part of this section, we find an upper bound for the number of facets of those polytopes $C$ for which every $C$-ball convex set is $k$-generated. It is also natural to estimate these numbers from below. Now, we consider the following problem: For a fixed integer $k \geq 2$, what is the maximum number $m=m(n, k)$ for which every convex $n$-polytope $C$ that has at most $m$ facets, also has the property that every $C$-ball convex set is $k$-generated? We have the following partial solution for this problem.

Theorem 10. Let $n \geq 3$.

(1) If $2 \leq k \leq n$, then there is a convex $n$-polytope $C$ having $n+k+2$ facets such that not every $C$-ball convex set is $k$-generated.

(2) If $k \geq 2$, and $C$ is any convex $n$-polytope having at most $n+k+1$ facets, then every $C$-ball convex set is $k$-generated. 
The example of a pentagon shows that the assertion in (2) fails for $n=$ $k=2$. It is easy to see that (2) also holds for $n=2$ and $k \geq 3$.

Proof. To prove (1), let $C$ be the convex $n$-polytope which is obtained from an $n$-simplex $S_{n}$ by intersecting it with $k$ closed half-spaces near $k$ vertices of $S_{n}$ so that the $k$ new facets are pairwise disjoint. Let $0<r<1$ arbitrary. Observe that for any set $T$ of $k$ points, $r C$ has a facet disjoint from $T$. Thus, by Lemma $6.2, r C$ is not the $C$-ball convex hull of at most $k$ points.

Next, we prove (2). Now assume that $C$ is a convex $n$-polytope of $n+k+1$ facets. Let $P$ be an arbitrary $C$-ball convex set of $\mathbb{R}^{n}, P \neq \mathbb{R}^{n}$. Then $P$ is a polytope of at most $n+k+1$ facets. We will assume that $C$ is a simple convex polytope. We may do so, since it is easy to see that for every $C$-ball convex set $P$ there is a sequence $\left\{P_{i}\right\}_{i=1}^{\infty}$ of simple convex $n$-polytopes such that every $P_{i}$ is a $C_{i}$-ball convex set, and $P_{i} \rightarrow P, C_{i} \rightarrow C$ in the Hausdorff metric, as $i \rightarrow \infty$, where $C_{i}$ is a convex $n$-polytope for every $i=1,2,3, \ldots$ Then any sequence of $k$ element subsets which span $P_{i}$ as a $C_{i}$-ball convex hull, for $i=1,2,3, \ldots$, have a subsequence whose elements converge to a $k$ element subset of $P$. Clearly, the $C$-ball convex hull of this set is $P$. By a similar limit argument, we may further assume that $\operatorname{dim} P=n, P$ is a simple polytope, and $P$ has exactly $n+k+1$ facets.

We need to show that there are $k$ points whose $C$-ball convex hull is $P$. By Lemma 6.2, it is sufficient to prove that there are $k$ vertices of $P$ such that for each facet $F$ of $C$ there is a translate $C+t$ of $C\left(t \in \mathbb{R}^{n}\right)$ that contains $P$ and for which $F+t$ contains at least one of the $k$ points. With the above assumption on the number of facets and dimension of $P$, it is equivalent to the existence of a set $T$ of $k$ points such that every facet contains at least one element of $T$.

Let $v$ be a vertex of $P$. Let $H$ be a hyperplane such that $v \notin H$ and $H$ is parallel to a supporting hyperplane $H^{\prime}$ of $P$ at $v$ for which $H^{\prime} \cap P=\{v\}$. Let $\pi$ be the central projection of $\mathbb{R}^{n} \backslash H^{\prime}$ to $H$ from the point $v$, that is $\pi(x)=\operatorname{aff}(v, x) \cap H$. Then $\pi(P)$ is bounded, in fact, it is an $(n-1)$ dimensional simplex $S_{n-1}$ since $P$ is a simple convex $n$-polytope. Consider the projection of the facets of $P$ under $\pi$. Then the images of those facets which contain $v$ are the facets of an $S_{n-1}$, we denote them by $\mathcal{F}=\left\{F_{1}, F_{2}, \ldots, F_{n}\right\}$. The images of the remaining $k+1$ facets form a tiling of $S_{n-1}$, we denote them by $\mathcal{A}=\left\{A_{1}, A_{2}, \ldots, A_{k+1}\right\}$. Clearly, every $A_{i}$ is an $(n-1)$-dimensional convex polytope. Denote by $V$ the vertex set of $S_{n-1}$.

Obviously, there are $k$ points that span $P$ as the $C$-ball convex hull of 
those points if and only if either there are $k-1$ points of $S_{n-1}$ such that every $A_{i}$ contains at least one of them, or there are $k$ points of $S_{n-1}$ such that every $A_{i}$ and $F_{j}$ contains at least one of them. If there are three elements of $\mathcal{A}$ having a common point, then such a set of $k-1$ points exists, just pick any point belonging to the three elements of $\mathcal{A}$ and one point from each remaining element of $\mathcal{A}$. So, from now on, we may assume that there is no common point of three elements of $\mathcal{A}$.

Since $\mathcal{A}$ contains more than one element, no $A_{i}$ contains all the vertices of $S_{n-1}$.

Now we show that there are two disjoint elements of $\mathcal{A}$ such that both contain at least one vertex of $S_{n-1}$. Let $A_{1}$ and $A_{2}$ be an intersecting pair, $A_{i} \cap V \neq \emptyset, i=1,2$. Consider an $(n-2)$-dimensional affine subspace $H_{12}$ that separates them in $H$. Then $A_{1} \cap A_{2}=H_{12} \cap S_{n-1}$ since $H_{12} \cap S_{n-1}$ is covered by $\mathcal{A}$ and it can not intersect any element of $\mathcal{A}$ distinct from $A_{1}$ and $A_{2}$ (otherwise, there would be a common point of $A_{1} \cap A_{2}$ and some $A_{i}$, for $i \neq 1,2)$. Thus we also obtain that $A_{1} \cap A_{2}$ is $(n-2)$-dimensional. Now, if $p \in A_{1}, q \in A_{2}$ are arbitrary points, then let $x=[p, q] \cap H_{12}$. We obtain $[p, x] \subseteq A_{1},[x, q] \subseteq A_{2}$, so $A_{1} \cup A_{2}$ is convex, and therefore it can not contain all vertices of $S_{n-1}$. So there is an $A_{j}, j \neq 1,2$, say $A_{3}$ such that $A_{3} \cap V \neq \emptyset$, and either $A_{1}$ or $A_{2}$, say $A_{1}$, is disjoint from $A_{3}$, because $A_{3}$ is disjoint from $H_{12}$. So we found two disjoint elements of $\mathcal{A}, A_{1}$ and $A_{3}$, even if $A_{1}$ and $A_{2}$ were intersecting.

Let us choose two disjoint elements of $\mathcal{A}$, say $A_{1}$ and $A_{2}$, such that $A_{i} \cap$ $V \neq \emptyset, i=1,2$. Since $S_{n-1}$ has $n \geq 3$ vertices, it follows that one of them, say $A_{1}$, contains at most $n-2$ vertices of $S_{n-1}$. Now, we pick a vertex $w$ in $A_{2} \cap V$ and an edge $E$ of $S_{n-1}$ connecting a vertex from $V \cap A_{1}$ and a vertex from $V \backslash\left(A_{1} \cup\{w\}\right) \neq \emptyset$. Furthermore, we may pick an $A_{i}$, say $A_{3}$, such that $E \cap A_{1} \cap A_{3} \neq \emptyset$, since $E$ intersects $A_{1}$ but also has points outside $A_{1}$. Finally, the desired $k$ points: Take a point $u \in E \cap A_{1} \cap A_{3}$, take $w \in A_{2}$, and take further $k-2$ points, one from each remaining $A_{i}$. As $u$ is contained in $E$, it is contained in each facet of $S_{n-1}$ but the two that do not contain $E$. These two facets contain $w$, and thus, every element of $\mathcal{F}$ contains at least one of the points. Since, clearly, the same holds for the elements of $\mathcal{A}$ as well, these points indeed satify the required property.

We ask the following question.

Problem 6.7. For any given $n \geq 3$ and $k \geq 2$, find a geometric characterization of those $n$-dimensional convex polytopes $C$ for which every $C$-ball 
convex set is $k$-generated. In particular, find a geometric characterization of those $n$-dimensional convex polytopes $C$ for which every $C$-ball convex set is a $C$-spindle.

\section{Stability of the operation $\mathrm{B}_{C}^{+}$and covering intersections of balls}

We consider the Levy-Markus-Gohberg-Boltyanski-Hadwiger Covering Problem (also know as the Illumination Problem) for two families of convex bodies, denoted by $\mathcal{D}(C)$ and $\tilde{\mathcal{D}}(C)$ (see Definition 7.1, and Remark 7.2), associated to any convex body $C$.

The covering number (see Definition 7.3) of sets of Euclidean constant width (members of $\mathcal{D}\left(B^{n}\right)$ ) has been studied extensively. One reason for its popularity is its connection to Borsuk's problem on partitioning convex sets into sets of smaller diameter. Weissbach [37] and Lassak [23] proved that the covering number of a set of Euclidean constant width in $\mathbb{R}^{3}$ is at most six. In [5], this result is extended to any set $K$ obtained as the intersection of Euclidean unit balls with the property that the set of the centers is of diameter at most one (members of $\tilde{\mathcal{D}}\left(B^{n}\right)$ ). Recently, further bounds on the covering number of sets in $\tilde{\mathcal{D}}\left(B^{n}\right)$ in dimensions $n=4,5$ and 6 have been found by Bezdek and Kiss [4]. The best general bound is due to Schramm [32] who proved that the covering number of a set of Euclidean constant width in $\mathbb{R}^{n}$ is at most $5 n^{3 / 2}(4+\log n)\left(\frac{3}{2}\right)^{n / 2}$. This result has been extended to members of $\tilde{\mathcal{D}}\left(B^{n}\right)$ as well, cf. Bezdek [2]. For surveys on covering (illumination) see Bezdek [1], and Martini and Soltan [25].

In this section, we study the stability of bounds on the covering number of convex sets in $\mathcal{D}(C)$ and in $\tilde{\mathcal{D}}(C)$. First, we prove that the operation $\mathbf{B}_{C}^{+}$is stable in a certain sense (Proposition 7.4), and then we deduce our stability results concerning covering numbers.

Definition 7.1. Let $C$ be a convex body in $\mathbb{R}^{n}$. Let

$$
\begin{gathered}
\mathcal{D}(C)=\left\{K \subset \mathbb{R}^{n}: K=\mathbf{B}_{C}^{+}(K)\right\}, \text { and } \\
\tilde{\mathcal{D}}(C)=\left\{K \subset \mathbb{R}^{n}: K=\mathbf{B}_{C}^{+}(X) \text { for some } X \subset \mathbb{R}^{n} \text { with } X \subseteq \mathbf{B}_{C}^{+}(X)\right\} .
\end{gathered}
$$

Remark 7.2. For any convex body $C$, we have $\mathcal{D}(C) \subseteq \tilde{\mathcal{D}}(C)$. Moreover, if $C=-C$ then $\mathcal{D}(C)$ is the family of diametrically maximal sets of diameter one in the Minkowski space (that is, finite dimensional real Banach space) with unit ball $C$. Since in the Euclidean space, a convex set is diametrically 
maximal if, and only if, it is of constant width, it follows that $\mathcal{D}\left(B^{n}\right)$ is the family of sets of Euclidean constant width one.

Definition 7.3. Let $K \in \mathbb{R}^{n}$ be a convex body. The covering number (also called the illumination number) $i(K)$ of $K$ is the minimum number of positive homothetic copies of $K$, with homothety ratio less than one, that cover $K$. For a family $\mathcal{F}$ of convex bodies, we set $i(\mathcal{F})=\max \{i(K): K \in \mathcal{F}\}$. We note that the illumination number is usually defined via the notion of illumination by directions (or light sources), which we do not follow here - the equivalence of those definitions with the one given here is well known, cf. [1].

The covering number is invariant under non-singular affine transformations, thus it is natural to use the Banach-Mazur distance to compare two convex bodies $K$ and $L$ :

$$
d(K, L)=\inf \{\lambda>0: K \subset T(L) \subset \lambda(K)\}
$$

where the infimum is taken over all non-singular affine transformations $T$. Recall that this distance is multiplicative (the triangle inequality holds with multiplication instead of addition) and the distance of a convex body from any non-singular affine image of itself is one.

We phrase, informally, the problem of the stability of the covering number in the following two manners.

Question 1. Fix a convex body $C$. If $K$ is 'close' to a set $L \in \mathcal{D}(C)$ (resp., to a set $L \in \tilde{\mathcal{D}}(C))$, does it follow that $i(K) \leq i(\mathcal{D}(C))$ (resp., $i(K) \leq$ $i(\tilde{\mathcal{D}}(C)))$ ?

Question 2. Fix a convex body $C$. If $D$ is 'close' to $C$, does it follow that $i(\mathcal{D}(D)) \leq i(\mathcal{D}(C))$ and $i(\tilde{\mathcal{D}}(D)) \leq i(\tilde{\mathcal{D}}(C)) ?$

Recall that $i(K)=n+1$ for any smooth convex body, while $i\left([0,1]^{n}\right)=2^{n}$ for the cube. It illustrates that the covering number, in general, may vary significantly along arbitrarily small perturbations. However, Theorems 11 and 12 provide positive answers to both questions.

Theorem 11. For every $n \in \mathbb{Z}^{+}$and for every convex body $C \subset \mathbb{R}^{n}$, there is a $\delta>1$ such that if $d(K, L)<\delta$ for a convex body $K$ in $\mathbb{R}^{n}$ and $L \in \mathcal{D}(C)$ (resp., $L \in \tilde{\mathcal{D}}(C)$ ) then $i(K) \leq i(\mathcal{D}(C))$ (resp., $i(K) \leq i(\tilde{\mathcal{D}}(C))$ ).

Theorem 12. For every $n \in \mathbb{Z}^{+}$and for every convex body $C \subset \mathbb{R}^{n}$, there is a $\delta>1$ such that if $d(C, D)<\delta$ for a convex body $D \subset \mathbb{R}^{n}$ then 
1. $i(\mathcal{D}(D)) \leq i(\mathcal{D}(C))$ and

2. $i(\tilde{\mathcal{D}}(D)) \leq i(\tilde{\mathcal{D}}(C))$.

The main tool in proving these results is the following observation that shows that the operation $\mathbf{B}_{C}^{+}$(and similarily, $\mathbf{B}_{C}^{-}$) is stable in a certain sense.

Proposition 7.4. Let $C_{1}, C_{2}, \ldots$ be a sequence of convex bodies in $\mathbb{R}^{n}$ converging to a convex body $C$ in the metric space of closed convex subsets of $\mathbb{R}^{n}$ equipped with the Hausdorff metric. Let $X_{1}, X_{2}, \ldots$ be a sequence of closed sets in $\mathbb{R}^{n}$ converging to a set $X$ such that the sequence $\mathbf{B}_{C_{i}}^{+}\left(X_{i}\right)$ also converges (to some set $K$ ). Assume that int $K \neq \emptyset$ or $\operatorname{int}\left(\mathbf{B}_{C}^{+}(X)\right) \neq \emptyset$. Then $K=\mathbf{B}_{C}^{+}(X)$.

Proof. First, we show that $\operatorname{int}\left(\mathbf{B}_{C}^{+}(X)\right) \subseteq K$. Let $u \in \mathbb{R}^{n} \backslash K$. Then, for infinitely many $k \in \mathbb{Z}$ there is a $q_{k} \in X_{k}$ such that $u \notin q_{k}+C$. By taking a subsequence, we may assume that the $q_{k}$ s converge to a point, say $q$. Clearly, $q \in X$, moreover $u \notin q+\operatorname{int} C$. Thus, $u \notin \operatorname{int}\left(\mathbf{B}_{C}^{+}(X)\right)$.

Next, we show that int $K \subseteq \mathbf{B}_{C}^{+}(X)$. Let $u \in \operatorname{int} K$. Then, there is a $\delta>0$ such that, for all sufficiently large $n \in \mathbb{Z}, u+\delta B^{n} \subset \mathbf{B}_{C_{n}}^{+}\left(X_{n}\right)$. It follows that, for all sufficiently large $n \in \mathbb{Z}, u \in \mathbf{B}_{C_{n}}^{+}(X)$. Hence, $u \in \mathbf{B}_{C}^{+}(X)$.

We leave it as an exercise to show that the condition $\operatorname{int}(\ldots) \neq \emptyset$ cannot be removed.

Let $\mathcal{K}^{n}$ denote the space of affine equivalence classes of convex bodies in $\mathbb{R}^{n}$ endowed with the Banach-Mazur distance. We show that $i: \mathcal{K}^{n} \rightarrow \mathbb{Z}^{+}$is upper semi-continuous:

Proposition 7.5. Let $K \in \mathcal{K}^{n}$. Then there is a $\delta>1$ such that $i(L) \leq i(K)$ for any $L \in \mathcal{K}^{n}$ with $d(K, L)<\delta$.

Proof. Let $m=i(K)$. Then $K$ is covered by $v_{1}+\lambda K, \ldots, v_{m}+\lambda K$ for some $0<\lambda<1$ and some translation vectors $v_{1}, \ldots, v_{m}$. Let $\delta=1 / \sqrt{\lambda}$. We may assume that $L \subseteq K \subseteq \delta L$. Then $L$ is covered by $v_{1}+\sqrt{\lambda} L, \ldots, v_{m}+\sqrt{\lambda} L$.

Remark 7.6. Let $C \subset \mathbb{R}^{n}$ be a convex body and $X \subset \mathbb{R}^{n}$ a set for which $X \subseteq \mathbf{B}_{C}^{+}(X)$. Then $\operatorname{int}\left(\mathbf{B}_{C}^{+}(X)\right) \neq \emptyset$. To see this, one may assume that $X$ is convex, and then show that any point in the relative interior of $X$ is an interior point of $\mathbf{B}_{C}^{+}(X)$. It follows that the members of $\mathcal{D}(C)$ and $\tilde{\mathcal{D}}(C)$ are bodies. 
Proof of Theorem 11. By the semi-continuity of $i$ on $\mathcal{K}^{n}$ (Proposition 7.5) and the compactness of $\mathcal{K}^{n}$, it is sufficient to show that $\tilde{\mathcal{D}}(C)$ and $\mathcal{D}(C)$ are closed subsets of $\mathcal{K}^{n}$. Let $K_{1}, K_{2}, \ldots$ be a convergent sequence of convex bodies in $\tilde{\mathcal{D}}(C)$. Then, by John's theorem, each one has an affine image $K_{i}^{\prime}$ such that $B^{n} \subseteq K_{i}^{\prime} \subseteq n B^{n}$. Now, $K_{1}^{\prime}, K_{2}^{\prime}, \ldots$ is a sequence of convex bodies in $\tilde{\mathcal{D}}(C)$ which is convergent with respect to the Hausdorff distance. By Proposition 7.4, the limit is also in $\tilde{\mathcal{D}}(C)$. The statement for $\mathcal{D}(C)$ easily follows.

Proof of Theorem 12. By Theorem 11, we need to prove that for any $\varepsilon>1$ there is a $\delta>1$ such that if $d(C, D)<\delta$ then for every $L \in \mathcal{D}(D)$ there is a $K \in \mathcal{D}(C)$ with $d(K, L)<\varepsilon$.

Let $D_{k}$ be a sequence of convex bodies in $\mathbb{R}^{n}$ such that $d\left(C, D_{k}\right)<1+\frac{1}{k}$ and let $X_{k}$ be a sequence of sets such that $L_{k}:=\mathbf{B}_{C_{k}}^{+}\left(X_{k}\right) \in \mathcal{D}\left(C_{k}\right)$. Suppose by contradiction that for each $L_{k}$, the closest member of $\mathcal{D}(C)$ is of distance at least $\mu>1$. By compactness, we may choose a convergent subsequence of the $X_{k}$ s. By taking a subsequence again, we may assume that the $L_{k}$ s converge, too. Now, by Proposition 7.4 (using John's position, as in the proof of Theorem 11), the limit of these $L_{k} \mathrm{~s}$ is a member of $\mathcal{D}(C)$, a contradiction.

Acknowledgements. We are grateful to Antal Joós and Steven Taschuk for the valuable conversations that we had with them on various topics covered in these notes.

[1] K. Bezdek, The illumination conjecture and its extensions, Period. Math. Hungar. 53 (2006) 59-69.

[2] K. Bezdek, Classical Topics in Discrete Geometry, CMS Books in Mathematics, Springer, to appear.

[3] K. Bezdek, R. Connelly, B. Csikós, On the perimeter of the intersection of congruent disks, Beiträge Algebra Geom. 47 (2006) 53-62.

[4] K. Bezdek, G. Kiss, On the X-ray number of almost smooth convex bodies and of convex bodies of constant width, Canad. Math. Bull. 52 (2009) 342-348.

[5] K. Bezdek, Z. Lángi, M. Naszódi, P. Papez, Ball-polyhedra, Discrete Comput. Geom. 38 (2007) 201-230. 
[6] K. Bezdek, M. Naszódi, Rigidity of ball-polyhedra in Euclidean 3-space, European J. Combin. 27 (2006) 255-268.

[7] L. Bieberbach, Zur Euklidischen Geometrie der Kreisbogendreiecke, Math. Ann. 130 (1955) 46-86.

[8] L. Bieberbach, Zwei Kongruenzsätze für Kreisbogendreiecke in der Euklidischen Ebene, Math. Ann. 190 (1970/1971) 97-118.

[9] H.G. Eggleston, Sets of constant width in finite dimensional Banach spaces, Israel J. Math. 3 (1965) 163-172.

[10] I. Fáry, E. Makai, Jr., Isoperimetry in variable metric, Studia Sci. Math. Hungar. 17 (1982) 143-158.

[11] S. Gołąb, Some metric problems of the geometry of Minkowski, Trav. Acad. Mines Cracovie 6 (1932) 1-79.

[12] B. Grünbaum, A proof of vázsonyi's conjecture, Bull. Research Couincil Israel A 6 (1956) 77-78.

[13] A. Heppes, Beweis einer Vermutung von A. Vázsonyi, Acta Math. Acad. Sci. Hungar. 7 (1956) 463-466.

[14] A. Heppes, P. Révész, A splitting problem of Borsuk, Mat. Lapok 7 (1956) 108-111.

[15] D.C. Kay, E.W. Womble, Axiomatic convexity theory and relationships between the Carathéodory, Helly, and Radon numbers, Pacific J. Math. 38 (1971) 471-485.

[16] W. Kubis, Separation properties of convexity spaces, J. Geom. 74 (2002) 110-119.

[17] Y. Kupitz, H. Martini, M. Perles, Finite sets in $\mathbb{R}^{d}$ with many diameters - a survey., Proceedings of the international conference on mathematics and its applications, ICMA-MU 2005, Bangkok, Thailand, December 15-17, 2005. Bangkok: Mahidol University. 91-112 (2005)., 2005.

[18] Y. Kupitz, H. Martini, B. Wegner, A linear-time construction of Reuleaux polygons, Beiträge Algebra Geom. 37 (1996) 415-427. 
[19] Y.S. Kupitz, H. Martini, M.A. Perles, Ball polytopes and the Vázsonyi problem, Acta Math. Hungar. 126 (2010) 99-163.

[20] Z. Lángi, M. Naszódi, Kirchberger-type theorems for separation by convex domains, Period. Math. Hungar. 57 (2008) 185-196.

[21] M. Lassak, Carathéodory's and Helly's dimensions of products of convexity structures, Colloq. Math. 46 (1982) 215-225.

[22] M. Lassak, On five points in a plane convex body pairwise in at least unit relative distances, in: Intuitive geometry (Szeged, 1991), volume 63 of Colloq. Math. Soc. János Bolyai, North-Holland, Amsterdam, 1994, pp. $245-247$.

[23] M. Lassak, Illumination of three-dimensional convex bodies of constant width, in: Proceedings of the 4th International Congress of Geometry (Thessaloniki, 1996), Giachoudis-Giapoulis, Thessaloniki, 1997, pp. 246-250.

[24] S.R. Lay, Separating two compact sets by a parallelotope, Proc. Amer. Math. Soc. 79 (1980) 279-284.

[25] H. Martini, V. Soltan, Combinatorial problems on the illumination of convex bodies, Aequationes Math. 57 (1999) 121-152.

[26] H. Martini, M. Spirova, On the circular hull property in normed planes, Acta Math. Hungar. 125 (2009) 275-285.

[27] M. Naszódi, S. Taschuk, On the transversal number and VC-dimension of families of positive homothets of a convex body, Discrete Math. 310 (2010) 77-82.

[28] J. Pach, P.K. Agarwal, Combinatorial geometry, Wiley-Interscience Series in Discrete Mathematics and Optimization, John Wiley \& Sons Inc., New York, 1995. A Wiley-Interscience Publication.

[29] J.R. Reay, Caratheodory theorems in convex product structures, Pacific J. Math. 35 (1970) 227-230.

[30] J.J. Schäffer, Inner diameter, perimeter, and girth of spheres, Math. Ann. 173 (1967), 59-79; addendum, ibid. 173 (1967) 79-82. 
[31] R. Schneider, Convex bodies: the Brunn-Minkowski theory, volume 44 of Encyclopedia of Mathematics and its Applications, Cambridge University Press, Cambridge, 1993.

[32] O. Schramm, Illuminating sets of constant width, Mathematika 35 (1988) 180-189.

[33] G. Sierksma, Carathéodory and Helly-numbers of convex-productstructures, Pacific J. Math. 61 (1975) 275-282.

[34] G. Sierksma, Extending a convexity space to an aligned space, Nederl. Akad. Wetensch. Indag. Math. 46 (1984) 429-435.

[35] S. Straszewicz, Sur un problème géométrique de P. Erdös, Bull. Acad. Polon. Sci. Cl. III. 5 (1957) 39-40, IV-V.

[36] M.L.J. van de Vel, Theory of convex structures, volume 50 of NorthHolland Mathematical Library, North-Holland Publishing Co., Amsterdam, 1993.

[37] B. Weißbach, Invariante Beleuchtung konvexer Körper, Beiträge Algebra Geom. 37 (1996) 9-15. 